\title{
Field theory of absorbing phase transitions with a non-diffusive conserved field
}

\author{
Romualdo Pastor-Satorras ${ }^{(1,2)}$ and Alessandro Vespignani ${ }^{(2)}$ \\ 1) Dept. de Física Fonamental, Facultat de Física, Universitat de Barcelona, Av. Diagonal 647, \\ 08028 Barcelona, Spain \\ 2) The Abdus Salam International Centre for Theoretical Physics (ICTP), P.O. Box 586, 34100 \\ Trieste, Italy
}

(October 24, 2018)

\begin{abstract}
We investigate the critical behavior of a reaction-diffusion system exhibiting a continuous absorbing-state phase transition. The reaction-diffusion system strictly conserves the total density of particles, represented as a non-diffusive conserved field, and allows an infinite number of absorbing configurations. Numerical results show that it belongs to a wide universality class that also includes stochastic sandpile models. We derive microscopically the field theory representing this universality class.
\end{abstract}

PACS numbers: 64.60.Ht, 05.70.Ln, 05.65.+b, 05.50.+q 
The directed percolation (DP) [] universality class is recognized as the canonical example of the critical behavior in the transition from an active to a single absorbing state. This universality class appears to be very robust with respect to microscopic modifications, and non-DP behavior emerges only in the presence of additional symmetries, such as in the case of symmetric absorbing states [2], long-range interactions [3], or infinitely many absorbing states [4].

Recently a new universality class of absorbing-state phase transitions (APT) [1] coupled to a non-diffusive conserved field has been identified [0] cal behavior of several models showing absorbing transitions with a dynamics that strictly conserves the density of particles, that is represented by a conserved static (non-diffusive) field. The models are tuned to criticality by varying the particle density in the initial state, and exhibit an infinite number of absorbing states. This universality class is particularly interesting because it embraces also the large group of stochastic sandpile models [6] (and in particular the Manna model [7]) which are the prototypical examples that illustrate the ideas of self-organized criticality (SOC) [8]. These are driven dissipative models in which sand (or energy) is injected into the system and dissipated through the boundaries, leading eventually to a stationary state. In the limit of infinitesimally slow external driving, the systems approach a critical state characterized by an avalanche-like response. Recently it has been pointed out that this critical state is equivalent to the absorbing phase transition present in the fixed energy case, that is, in automata that consider the same microscopic rules defining the sandpile dynamics, but without driving nor dissipation [9]11].

The numerical evidence for the existence of such a general universality class [5] is corroborated by the observation that all the models analyzed share the same structure and basic symmetries; namely, a conserved and static non-critical field dynamically coupled to a non-conserved order parameter field, identified as the density of the active particles. These observations have led to the conjecture that, in absence of additional symmetries, all stochastic models with an infinite number of absorbing states in which the order parameter evolution is coupled to a non-diffusive conserved field define a unique universality class [5]. 
In this Letter, we study the non-diffusive field limit for the two species reaction-diffusion model introduced in Ref. [12] (see also Ref. [13]). In this limit the model has a phase transition with infinitely many absorbing states and it conserves the total number of particles, that is associated to a non-diffusive conserved field. We present extensive numerical simulations of the model in two and three dimensions, and determine the full set of critical exponents. The obtained values are compatible with the new universality class conjectured in Ref. [5]. This definitely shows the existence of a very broad universality class that includes reactiondiffusion processes, stochastic sandpile models, and lattice gases with the same symmetry properties. For the present reaction-diffusion model, it is possible to derive microscopically a field theory (FT) description. The resulting action and Langevin equations exhibit the basic symmetries that characterize this universality class, and represent the first microscopic derivation of a FT for sandpile models. Notably, the resulting FT description recovers a phenomenological Langevin approach proposed for stochastic sandpiles [9, 10]. The analysis provided here is a very promising path for a coherent description of several nonequilibrium critical phenomena now rationalized in a single universality class.

We consider the two-component reaction-diffusion process identified by the following set of reaction equations:

$$
\begin{gathered}
B \rightarrow A \quad \text { with rate } k_{1} . \\
B+A \rightarrow 2 B \quad \text { with rate } k_{2},
\end{gathered}
$$

In this system, $B$ particles diffuse with diffusion rate $D_{B} \equiv D$, and $\mathrm{A}$ particles do not diffuse, that is, $D_{A}=0$. This corresponds to the limit $D_{A} \rightarrow 0$ of the model introduced in Ref. [12]. From the rate Eqs. (11) and (2), it is clear that the dynamics conserves the total density of particles $\rho=\rho_{A}+\rho_{B}$, where $\rho_{i}$ is the density of component $i=A, B$. In this conserved reaction-diffusion model, the only dynamics is due to $B$ particles, that we can identify as active particles. $A$ particles do not diffuse and cannot generate spontaneously $B$ particles. More specifically, $A$ particles can only move via the motion of $B$ particles that later on transform into $A$ because of Eq. (2). In absence of $B$ particles, $\rho_{A}$ can be considered 
a static field. This implies that any configuration devoid of $B$ particles is an absorbing state in which the system is trapped forever.

It is easy to see [12] that the reaction-diffusion process defined by Eqs. (11) and (2) exhibits a phase transition from an active to an absorbing phase for a non-trivial value of the total particle density $\rho=\rho_{c}$, which acts as the control parameter. The critical value $\rho_{c}$ depends upon the reaction rates $k_{1}, k_{2}$. The nature of this phase transition (whether it is first or second order) for $D_{A} \neq 0$ appears to be determined by the ratio between $D_{B}$ and $D_{A}$ [12]; the static field case $\left(D_{A}=0\right)$, on the other hand, has never been explored. It is clear that the static field conserved reaction-diffusion (SFCRD) model allows, for any density $\rho$, an infinite number (in the thermodynamic limit) of absorbing configurations, in which there are no $B$ particles. This is the key difference with respect to the case in which $D_{A} \neq 0$, as in Ref. [12]. In the latter case a configuration devoid of $B$ particles consists of many diffusing $A$ particles, jumping from site to site. In the long run, all particles can visit all sites, and therefore, in a statistical sense, all configuration with a fixed number of $A$ 's are equivalent and the absorbing state can be considered statistically unique [14].

The present SFCRD model seems to possess all the required symmetry (stochastic dynamics, many absorbing states, static conserved field) for being part of the universality class conjectured in Ref. [5]. In order to test this possibility, we have performed numerical simulations of the model in a $d$-dimensional hypercubic lattice with $N=L^{d}$ sites. Each site can store any number of $A$ and $B$ particles; that is, our model can be represented by bosonic variables. Initial conditions are generated by randomly placing $N \rho_{A}^{(0)}$ particles $A$ and $N \rho_{B}^{(0)}$ particles $B$, corresponding to a particle density $\rho=\rho_{A}^{(0)}+\rho_{B}^{(0)}$. The results are independent of the particular initial ratio $\rho_{A}^{(0)} / \rho_{B}^{(0)}$, apart from very early time transients. The dynamics proceeds in parallel. Each time step, we update the lattice according to the following rules. a) Diffusion: on each lattice site, each $B$ particles moves into a randomly chosen nearest neighbor site. b) After all sites have been updated for diffusion, we perform the reactions: i) On each lattice site, each $B$ particle is turned into an $A$ particle with probability $r_{1}$. ii) At the same time, each $A$ particle becomes a $B$ particle with probability $1-\left(1-r_{2}\right)^{n_{B}}$, 
where $n_{B}$ is the total number of $B$ particles in that site. This corresponds to the average probability for an $A$ particle of being involved in the reaction (2) with any of the $B$ particles present on the same site. The probabilities $r_{1}$ and $r_{2}$ are related to the reaction rates $k_{1}$ and $k_{2}$ defined in Eqs. (11) and (22). The order parameter of the system is $\rho_{B}$, measuring the density of dynamical entities. For small initial densities $\rho$, the system will very likely fall into an absorbing configurations with only frozen $A$ particles. For large densities, the system reaches a stationary active state with $\rho_{B} \neq 0$.

As we vary $\rho$, the system exhibits a continuous transition separating an absorbing phase from an active phase at a critical point $\rho_{c}$. The order parameter is null for $\rho<\rho_{c}$, and follows a power law $\rho_{B} \sim\left(\rho-\rho_{c}\right)^{\beta}$, for $\rho \geq \rho_{c}$. The system correlation length $\xi$ and time $\tau$, that define the exponential relaxation of space and time correlation functions, both diverge as $\rho \rightarrow \rho_{c}$ [1]. In the critical region the system is characterized by a power law behavior, namely $\xi \sim\left|\rho-\rho_{c}\right|^{-\nu_{\perp}}$ and $\tau \sim\left|\rho-\rho_{c}\right|^{-\nu_{\|}}$. The dynamical critical exponent is defined as $\tau \sim \xi^{z}$, with $z=\nu_{\|} / \nu_{\perp}$. These exponents fully determine the critical behavior of the stationary state of the model (see Ref. [1]).

We have studied the steady-state properties of the model in $d=2$ and 3 , by performing numerical simulations for systems with size ranging up to $L=512$ and $L=125$, respectively. Averages were performed over $10^{4}-10^{5}$ independent initial configurations. The values considered for the rates $r_{i}$ are $r_{1}=0.1$ and $r_{2}=0.5$ in $d=2$, and $r_{1}=0.4$ and $r_{2}=0.5$ in $d=3$. From the finite-size scaling analysis for absorbing phase transitions [1] we obtain the critical point $\left(\rho_{c}=0.3226(1)\right.$ in $d=2$ and $\rho_{c}=0.95215(15)$ in $\left.d=3\right)$ and the complete set of critical exponents. A detailed presentation of these results will be reported elsewhere. In Fig. [1 we show as an example the order parameter behavior with respect to the control parameter $\Delta=\rho-\rho_{c}$, from which it is possible to calculate directly the $\beta$ exponent. The results obtained in $d=2$ and 3 are reported in Tables I and II and compared with the Manna sandpile model in the respective dimension.

In APT it is possible to obtain more information on the critical state by studying the evolution (spread) of activity in systems which start close to an absorbing configuration 15. 
In each spreading simulation, a small perturbation is added to an absorbing configuration. It is then possible to measure the spatially integrated activity $N(t)$, averaged over all runs, and the survival probability $P(t)$ of the activity after $t$ time steps. Only at the critical point we have power law behavior for these magnitudes. In the case of many absorbing states, the choice of the initial absorbing state is not unique [16]. There are several methods to perform spreading exponents in this case, and we have followed the technique outlined in Ref. [0]. This procedure amounts to the study of critical spreading with the so-called "natural initial conditions" at $\rho=\rho_{c}$ [16]. The probability distribution $P_{s}(s)$ of having a spreading event involving $s$ sites, as well as the the quantities $N(t)$ and $P(t)$ can thus be measured. At criticality, the only characteristic length is the system size $L$, and we can write the scaling forms $P_{s}(s)=s^{-\tau_{s}} h\left(s / L^{D}\right), N(t)=t^{\eta} f\left(t / L^{z}\right)$, and $P(t)=t^{-\delta} g\left(t / L^{z}\right)$ 15. The scaling functions $f(x), g(x)$ and $h(x)$ are decreasing exponentially for $x \gg 1$, and we have considered that the spreading characteristic time and size are scaling as $L^{z}$ and $L^{D}$, respectively. In this case simulations were performed for systems of size up to $L=1024$ in $d=2$ and $L=200$ in $d=3$, averaging over at least $5 \times 10^{6}$ spreading experiments. The new scaling exponents $\tau_{s}, D, \delta$, and $\eta$ are measured using the now standard moment analysis technique [17, [1] , and a consistency check is executed by performing a data collapse analysis. The resulting exponents are summarized in Tables I and II. As a further consistency check of our results, we have checked that our exponents fulfill all scaling and hyper-scaling relations in standard APT. Despite the apparent diversity in the dynamical rules, we can safely include the SFCRD model and the Manna model in the same universality class. The reported numerical values provide striking evidence for a single universality class, and a further check of the conjecture in Ref. [5].

From a theoretical point of view, the SFCRD allows the microscopic construction of a field theory description that will represent also the critical behavior of all models belonging to the same universality class. The construction of the FT follows the standard steps outlined in the work of Doi, Peliti, and also in Lee and Cardy [21]. This technique consists in recasting the master equation implicit in Eqs. (11) and (2) into a "second quantized form" via a set of 
creation and annihilation bosonic operators for particles $A$ and $B$ on each site. At this point it is possible to map the solution of the master equation in a path integral on the density fields weighted by the exponential of a functional action $S$ [21]. In our case, we can quote the elegant results of Ref. [12], just considering that we have $D_{A}=0$. The action of the FT is thus

$$
\begin{aligned}
S & =\int d x d t\left\{\bar{\psi}\left[\partial_{t}+\left(r-D \nabla^{2}\right)\right] \psi+\bar{\phi}\left[\partial_{t} \phi-\lambda \nabla^{2} \psi\right]\right. \\
& +u_{1} \bar{\psi} \psi(\psi-\bar{\psi})+u_{2} \bar{\psi} \psi(\phi+\bar{\phi}) \\
& \left.+v_{1} \bar{\psi}^{2} \psi^{2}+v_{2} \bar{\psi} \psi(\psi \bar{\phi}-\bar{\psi} \phi)+v_{3} \bar{\psi} \psi \phi \bar{\phi}\right\}
\end{aligned}
$$

where $\psi$ and $\phi$ are auxiliary fields, defined such that their average values coincide with the average density of $B$ particles and the total density of particles, respectively, $\bar{\psi}$ and $\bar{\phi}$ are response fields, and the coupling constants are related to the reaction rates $k_{i}$. Namely, $D$ represents the diffusion coefficient of $B$ particles, $\lambda$ is initially also proportional to $D$, and $r$ is the critical parameter that plays the role of the mass in the FT and is related to the difference of the total density with respect to the critical density $\rho_{c}$. The critical point in the mean-field theory is of course at $r_{c}=0$. By standard power-counting analysis, one realizes that the reduced couplings $u_{i} / D$ have critical dimension $d_{c}^{(1)}=4$, while the couplings $v_{i} / D$ have on their part $d_{c}^{(2)}=2$. This means that when applying the renormalization group $(\mathrm{RG})$ and performing a perturbative expansion around the critical dimension 4, one could in principle drop all the couplings $v_{i}$ [22]. The critical parameter of this theory is the density of active sites $\psi$, while $\phi$ serves just to propagate interactions. We can exploit some symmetry considerations of the FT to relate the physics of the system to the corresponding analytical description. In fact, by neglecting irrelevant terms in the power counting analysis, the action (3) is invariant under the shift transformation

$$
\phi \rightarrow \phi^{\prime}=\phi+\delta, \quad r \rightarrow r^{\prime}=r-u_{2} \delta
$$

where $\delta$ is any constant. This symmetry has a very intuitive meaning: If we increase everywhere the density of the system by an amount $\delta$, we must be closer to the critical point 
by an amount proportional to $\delta$. In other words, this symmetry represents the conserved nature of the system. It is also interesting to write the set of corresponding Langevin equations (up to irrelevant terms) by integrating out the response fields $\bar{\psi}, \bar{\phi}$ in the action $S:$

$$
\begin{aligned}
& \partial_{t} \psi=D \nabla^{2} \psi-r \psi-u_{1} \psi^{2}-u_{2} \psi \phi+\eta_{\psi}, \\
& \partial_{t} \phi=\lambda \nabla^{2} \psi+\eta_{\phi} .
\end{aligned}
$$

Here, $\eta_{\psi}$ and $\eta_{\phi}$ are noise term with zero mean and correlations $\left\langle\eta_{\psi}(x, t) \eta_{\psi}\left(x^{\prime}, t^{\prime}\right)\right\rangle=$ $2 u_{1} \psi(x, t) \delta\left(x-x^{\prime}\right) \delta\left(t-t^{\prime}\right),\left\langle\eta_{\psi}(x, t) \eta_{\phi}\left(x^{\prime}, t^{\prime}\right)\right\rangle=-u_{2} \psi(x, t) \delta\left(x-x^{\prime}\right) \delta\left(t-t^{\prime}\right)$ and $\left\langle\eta_{\phi}(x, t) \eta_{\phi}\left(x^{\prime}, t^{\prime}\right)\right\rangle=0$. The noise terms have a multiplicative nature [23], that is the standard form in APT. Note that the $v_{i}$ couplings contribute to the noises correlations with higher order terms. These equations have a very clear physical interpretation. The field $\phi$ is conserved and static, i.e., it only diffuses via the activity of $B$ particles, represented by the field $\psi$ [24]. On its turn, the field $\psi$ is locally coupled to the field $\phi$, but is non-conserved. Noticeably, this set of equations recovers the Langevin description that has been proposed on a phenomenological level for stochastic sandpiles [9,[10], with the extra infromation of the cross-correlation term $\left\langle\eta_{\psi} \eta_{\phi}\right\rangle$. Indeed, the sandpile model has the same basic symmetries of the present reaction-diffusion model, once the local density field $\rho$ is replaced by the local sand-grain (energy) density and the order parameter is identified with the density of toppling sites field [9:10]. It is then natural to expect that the very same basic structure is reflected in a unique theoretical description. This observation substantiates the existence of a common universality class that embraces stochastic sandpiles, conserved lattice gases and reaction diffusion systems with many absorbing states.

The complete RG analysis of the field theory would allow to extract estimates for the critical exponents to compare with simulations in $d=2$ and 3. Unfortunately, several severe technical problems are encountered in this case. In general, as pointed out in Ref. [12], the couplings $v_{i}$ become relevant and should be taken into account in the RG analysis. The importance of the couplings $v_{i}$ can be argued by the change of the energy shift symmetry 
form, Eq. (4), in the case of the full action, Eq. (3). Second, and more important, is the presence of the singular bare propagator for the field $\phi$, that cannot be regularized by adding a mass term $m^{2} \phi \bar{\phi}$, since it will obviously break the symmetry (四). This singular propagator gives rise to divergences in the $\mathrm{RG}$ perturbative expansions, and the results of Ref. [12] cannot be extended "tout-court" to the limit $D_{A} \rightarrow 0$. In particular, some Feynman diagrams in the $\epsilon$-expansion presented in Refs. [12,13] are proportional to $1 / D_{A}$. Hence, the limit $D_{A} \rightarrow 0$ in the theory with $D_{A} \neq 0$ is non-analytic; any infinitesimal amount of diffusion in the energy field renormalizes to a finite value, and definitely changes the universality class of the model. Work is in progress to provide a suitable regularization that will allow an $\epsilon$-expansion calculation of the FT critical exponents.

This work has been supported by the European Network under Contract No. ERBFM-

RXCT980183. We thank D. Dhar, R. Dickman, P. Grassberger, H. J. Hilhorst, M.A. Muñoz, F. van Wijland and S. Zapperi, for helpful comments and discussions. 


\section{REFERENCES}

[1] J. Marro and R. Dickman, Nonequilibrium phase transitions in lattice models (Cambridge University Press, Cambridge, 1999).

[2] J. L. Cardy and U. Täuber, Phys. Rev. Lett. 77, 4780 (1996).

[3] H. K. Janssen, K. Oerding, F. van Wijland, and H. J. Hilhorst, European Phys. J. B 7, $137,(1999)$.

[4] E. V. Albano, J. Phys. A: Math. Gen. 25, 2557 (1992); I. Jensen, Phys. Rev. Lett. 70, 1465 (1993); M. A. Muñoz, G. Grinstein, R. Dickman, and R. Livi, Phys. Rev. Lett. 76, 451 (1996).

[5] M. Rossi, R. Pastor-Satorras, and A. Vespignani, e-print cond-mat/0004242.

[6] For a review see: H. J. Jensen, Self-Organized Criticality (Cambridge University Press, Cambridge, 1998).

[7] S. S. Manna, J. Phys. A 24, L363 (1991); D. Dhar, Physica A 263, 4 (1999).

[8] P. Bak, C. Tang, and K. Wiesenfeld, Phys. Rev. Lett. 59, 381 (1987).

[9] R. Dickman, A. Vespignani, and S. Zapperi, Phys. Rev. E 57, 5095 (1998); A. Vespignani, R. Dickman, M. A. Muñoz, and S. Zapperi, Phys. Rev. Lett.81, 5676 (1998). R. Dickman, M. A. Muñoz, A. Vespignani, and S. Zapperi, cond-mat/9910454.

[10] A. Vespignani, R. Dickman, M. A. Muñoz, and S. Zapperi, e-print cond-mat/0003285.

[11] The connection between SOC and APT has been also discussed for the Bak-Sneppen model, M. Paczuski, S. Maslov, and B. Bak, Europhys. Lett. 27, 97 (1994); Erratum 28, 295 (1994); P. Grassberger, Phys. Lett. A 200, 277 (1995).

[12] F. van Wijland, K. Oerding, and H. J. Hilhorst, Physica A 251, 179 (1998).

[13] R. Kree, B. Schaub, and B. Schmittmann, Phys. Rev. A 39, 2214 (1989). 
[14] In this sense, the model defined in Ref. [12] is similar to the threshold transfer process introduced by J. F. F. Mendes, R. Dickman, M. Henkel, and M. C. Marques, J. Phys. A: Math. Gen., 273019 (1994).

[15] P. Grassberger and A. de la Torre, Ann. Phys. (N.Y.) 122, 373 (1979).

[16] I. Jensen and R. Dickman, Phys. Rev. E 48, 1710 (1993).

[17] M. De Menech, A. L. Stella, and C. Tebaldi, Phys. Rev. E 58, R2677 (1998); C. Tebaldi, M. De Menech, and A. L. Stella, Phys. Rev. Lett 83, 3952 (1999);

[18] A. Chessa, A. Vespignani, and S. Zapperi, Comput. Phys. Comm. 121-122, 299 (1999); S. Lübeck, Phys. Rev. E 61, 204 (2000).

[19] K. Nakanishi and K. Sneppen, Phys. Rev. E 55, 4012 (1997).

[20] E, Milshtein, O. Biham, and S. Solomon, Phys. Rev. E 58, 303 (1998).

[21] M. Doi, J. Phys. A9, 1465 (1976); L. Peliti, J. Physique 46, 1469 (1985); B. P. Lee and J. Cardy, J. Stat. Phys. A 80, 971 (1995).

[22] In spite of the naive power counting analysis, the irrelevance of all terms must be checked on the grounds of a full RG analysis.

[23] J. L. Cardy and R. L. Sugar, J. Phys. A: Math. Gen. 13, L423 (1980); H. K. Janssen, Z. Phys. B 42, 151 (1981).

[24] It is possible to show that the present noise term $\eta_{\phi}$ is equivalent to a conserved noise; i.e., it generates the same diagrams in a perturbative expansion. After M.A. Muñoz and F.van Wijland, private communications. 


\section{TABLES}

\begin{tabular}{lccccc}
\hline \hline & \multicolumn{5}{c}{ Steady state exponents } \\
\cline { 2 - 5 } & $\beta$ & $\beta / \nu_{\perp}$ & $\nu_{\perp}$ & $z$ & $\nu_{\|}$ \\
\hline SFCRD & $0.65(1)$ & $0.78(2)$ & $0.83(3)$ & $1.55(5)$ & $1.29(8)$ \\
Manna & $0.64(1)$ & $0.78(2)$ & $0.82(3)$ & $1.57(4)$ & $1.29(8)$ \\
\hline \hline & & & Spreading exponents & \\
\cline { 2 - 6 } & $\tau_{s}$ & $D$ & $z$ & $\eta$ & $0.50(2)$ \\
SFCRD & $1.27(1)$ & $2.75(1)$ & $1.54(2)$ & $0.29(2)$ & $0.48(2)$ \\
Manna & $1.28(1)$ & $2.76(1)$ & $1.55(1)$ & $0.30(3)$ & \\
\hline \hline
\end{tabular}

TABLE I. Critical exponents for spreading and steady state experiments in $d=2$. Figures in parenthesis indicate the statistical uncertainty in the last digit. Manna exponents from Refs. [5, 10, 18,20$]$.

\begin{tabular}{|c|c|c|c|c|c|}
\hline & \multicolumn{5}{|c|}{ Steady state exponents } \\
\hline & $\beta$ & $\beta / \nu_{\perp}$ & $\nu_{\perp}$ & $z$ & $\nu_{\|}$ \\
\hline SFCRD & $0.86(2)$ & $1.39(4)$ & $0.62(3)$ & $1.80(5)$ & $1.12(8)$ \\
\hline \multirow[t]{3}{*}{ Manna } & $0.84(2)$ & $1.40(2)$ & $0.60(3)$ & $1.80(5)$ & $1.08(8)$ \\
\hline & \multicolumn{5}{|c|}{ Spreading exponents } \\
\hline & $\tau_{s}$ & $D$ & $z$ & $\eta$ & $\delta$ \\
\hline SFCRD & $1.41(1)$ & $3.32(1)$ & $1.74(2)$ & $0.16(1)$ & $0.76(2)$ \\
\hline Manna & $1.43(1)$ & $3.31(1)$ & $1.75(2)$ & $0.16(2)$ & $0.75(2)$ \\
\hline
\end{tabular}

TABLE II. Critical exponents for spreading and steady state experiments in $d=3$. Figures in parenthesis indicate the statistical uncertainty in the last digit. Manna exponents from Refs. [5, 10, 18, 20]. 


\section{FIGURES}

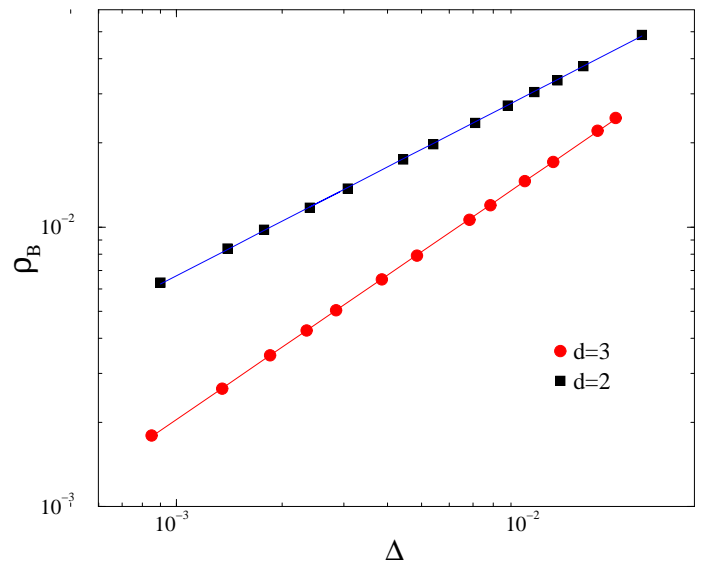

FIG. 1. Order parameter behavior (stationary density of $B$ particles) as a function of $\Delta=\rho-\rho_{c}$ for the reaction diffusion model in $d=2$ and 3 . The slope of the straight lines is $\beta=0.65$ in $d=2$ and $\beta=0.86$ in $d=3$. 